\title{
Rheumatic Diseases During Pregnancy
}

\author{
Gebelikte Romatizmal Hastalıklar
}

\author{
Rahman Yavuz
}

Sakarya Provincial Directorate Of Health, Maltepe Family Healthcare Center, Sakarya, Turrkey

\section{Abstract}

Pregnancy induces immunologic changes that may differentially impact rheumatic disorders. The effects of pregnancy on rheumatic diseases vary by condition. The systemic rheumatic illnesses commonly complicating

Keywords: Pregnancy, rheumatic disease, clinical approach pregnancy are systemic lupus erythematosus (SLE), antiphospholipid syndrome (APS), rheumatoid arthritis (RA), scleroderma.

\section{Introduction}

Pregnancy is an important condition that can affect and be affected by rheumatic disease. The effects of pregnancy on rheumatic diseases vary by condition. Rheumatoid arthritis, lupus and antiphospholipid syndrome typically are modified by pregnancy. For instance, symptoms of RA almost invariably improve in pregnant patients frequently resulting in a reduced need for medications. Most rheumatic diseases that are well controlled prior to pregnancy do not deteriorate in pregnancy, providing that the patient continues with appropriate disease-modifying therapy. Some patients with inflammatory arthritis go in to remission during pregnancy. $\mathrm{Pa}$ tients with renal involvement may be at increased risk of disease flare. During pregnancy it is essential to monitor all aspects of disease activity such as renal involvement, as well as pregnancy particularly in patients with syste- mic lupus erythematozus, antiphospholipid syndrome and systemic sclerosis ${ }^{1}$.

\section{Physiologic and immunologic changes in preg- nancy}

The female sex hormones play an important role in the aetiology and pathophysiology of chronic immune/inflammatory diseases. Pregnancy is a state of high concentration of sex hormones and cross-talk between mother and fetus. Oestrogens are generally considered as enhancers of cell proliferation and humoral immune responses, whereas androgens have opposite effects. Throughout pregnancy, the hormonal, biochemical and immunological equilibrium in the mother changes related to the stage of pregnancy ${ }^{2}$. Increased blood volume with a resultant physiologic anemia occurs ${ }^{3}$; thrombophilia and increased co-aguability likewise happen ${ }^{4}$. Muscu- 
loskeletal pain can be difficult to interpret as pregnancy itself leads to joint and skeletal complaints. Physiologic changes that happen with pregnancy can cause signs and symptoms difficult to differentiate from rheumatologic disease flares ${ }^{5}$. Furthermore, laboratory testing typically used to evaluate rheumatologic disease activity may be challenging to interpret during pregnancy. For example, in healthy pregnancies the erythrocyte sedimentation rate and the synthesis of complement components both increase and therefore these tests may not be good markers for disease activity ${ }^{6}$.

Pregnancy is a time of immunologic change. The increase of free steroid hormones including glucocorticoids, progesterones and oestrogens, induces changes in functions of immunocompetent cells such as B cells, T cells and monocytes ${ }^{7}$. Cytokines are important mediators involved in the successful outcome of pregnancy. One of the most important immunological modifications during pregnancy is the Thelper1(Th1)/Thelper2(Th2) shift due to the progressive increase of progesterone and estrogens during pregnancy which reach their peak-level in the third trimester of gestation. At high levels estrogens seem mainly to suppress Th1 cytokines and stimulate Th2-mediated immunological responses as well as antibody production ${ }^{8}$.

Both Th1 and Th2 type cytokines play a role at different stages of pregnancy and are adapted to the localization and function of cells and tissues. In rheumatic diseases with a predominance of a Th1 immune response a shift to a Th2 type immune response during pregnancy has been regarded as beneficial. Studies of pregnant patients with RA and systemic lupus erythematosus SLE have shown a cytokine expression similar to that found in healthy pregnant women. Significant differences were present only for a few cytokines and seemed related to the activity of the underlying disease. Interestingly, a gestational increase of cytokine inhibitors interleukin 1 receptor antagonist and soluble tumor necrosis factor receptor in the circulation corresponded to low disease activity in $\mathrm{RA}^{9}$.

\section{Rheumatic diseases in pregnancy}

a. Systemic lupus erythematosus

SLE is a multi-system autoimmune connective tissue disorder that primarily affects women of childbearing age. Typical clinical symptoms of SLE include fatigue, fever, arthritis, a photosensitive rash, serositis, Raynaud phenomenon, glomerulonephritis, vasculitis and hematologic abnormalities. Prevalence rates in SLE are estimated to be 51 per 100,000 in the United States ${ }^{10}$. Estimated incidence rates in North America, South America, and Europe range from 2 to 8 per 100,000 per year ${ }^{9}$. Women are affected nine times more frequently than men ${ }^{11}$.

Due to the nature of the disease and the increased risk of the disease flaring up during pregnancy women with SLE have previously often been advised to avoid pregnancy ${ }^{12}$. Some studies report exacerbation of SLE during pregnancy ${ }^{13}$ and the others have not reported increased flares $^{14}$. The Hopkins Lupus Pregnancy Cohort have shown an increase in renal flares in pregnant SLE patients and a Canadian prospective study found changes in renal disease activity and deterioration in renal function in pregnant patients similar to those which occur in nonpregnant patients with lupus nephritis ${ }^{15}$. When compared to other women, SLE patients are at increased risk for maternal death, preeclampsia, preterm labor, thrombosis, infection, unplanned cesarean section and hematologic complications during pregnancy. There are also increased risks to infants of women with SLE including low birth weight, fetal loss, and prematurity. The data obtained from the 'A National Study of the Complications of Lupus in Pregnancy' using the Nationwide Inpatient Sample from 2000 to 2003 and compared maternal and pregnancy complications for all pregnancy-related admissions for women with and without SLE. In the same study was found to women with SLE were more likely to have other medical conditions such as hypertension, diabetes, thrombophilia, the risks for thrombosis, infection, thrombocytopenia, and transfusion were each approximately 3-7-fold higher for women with SLE and had a higher risk for cesarean sections, preterm labor, preeclampsia than other women ${ }^{16}$. 
Studies indicating an increase in flares during pregnancy and 3 months postpartum when compared with nonpregnant lupus patients in a 12-month period ${ }^{17}$. Flares occur during all trimesters and often also occur postpartum. The frequency of flares ranges between 7 and $33 \%$ in women who have been in remission for at least 6 months prior to conception but flares occur in up to $61-67 \%$ of patients if the disease was active at conception18. If the disease is inactive at conception then the risk of flares is reduced in comparison with both those who had active disease at conception and the nonpregnant controls ${ }^{19}$. Signs and symptoms of exacerbation of SLE are including cytopenias, fever not due to infection, increased inflammatory rash often sparing the nasolabial folds, pleuritic chest pain, pericarditis. Increased creatinine or proteinuria compared with baseline or active urinary sediment. Any signs of an SLE flare should be aggressively treated with the lowest possible dose of glucocorticoids needed to control the disease. Corticosteroids are used to control active lupus during pregnancy. The fetus is protected from the effect of prednisone or hydrocortisone because of the placental enzyme 11 beta dehydrogenase. Prednisolone is a little crosses the placenta in doses up to $20 \mathrm{mg}$ daily so that adrenal neonatal suppression is rare, and it is important to maintain the minimum dose as with doses above $10 \mathrm{mg} / \mathrm{d}$, steroids can associated with an increased risk of preeclampsia, pregnancy induced hypertension, gestational diabetes, possible premature rupture of membranes ${ }^{20}$

Maternal thrombocytopenia, renal disease, cardiopulmonary disease may affect the maternal and fetal health. Thrombocytopenia is defined as a platelet count of less than $150,000 / \mu L^{21}$ and measuring the value of 100,000 to $150,000 / \mu \mathrm{L}$ are considered mildly depressed, 50,000 to $100,000 / \mu \mathrm{L}$ are moderately depressed and less than $50,000 / \mu \mathrm{L}$ are severely depressed can be defined $\mathrm{as}^{22}$. Thrombocytopenia complicates up to $10 \%$ of all pregnancies and occurring during pregnancy is a common problem and may have many causes such as gestational thrombocytopenia, acute fatty liver of pregnancy, SLE, preeclampsia, antiphospholipid antibodies, HELLP syndrome, thrombotic thrombocytopenic purpu$\mathrm{ra}^{23}$. Approximately $25 \%$ of pregnancies with SLE are complicated by hypertensive disorders and a higher rate of caesarean sections than in the control population ${ }^{24}$. The outcome of hypertension in pregnancy is affected by multiple factors including gestational age at onset, severity of disease and the presence of comorbid conditions such as preexisting hypertension, diabetes mellitus, renal disease, thrombophilia ${ }^{25}$. Adverse outcomes related to hypertension in pregnancy can be divided into short-term versus long-term complications. While shortterm complications can be further subgrouped into maternal and fetal complications, long-term outcomes are mainly maternal. There is approximately one maternal death due to preeclampsia-eclampsia per 100,000 live births with a case-fatality rate of 6.4 deaths per 10,000 cases $^{26}$.

One of the major complications of SLE is lupus nephritis (LN). During the second half of pregnancy significant proteinuria and hypertension develop in up to $25 \%$ of women with SLE but this may be due to either preeclampsia or lupus nephritis. The activity of LN at conception impacted on fetal losses which ranged between approximately 25 and $57 \%$ in women with active LN vs ${ }^{8-12} .5 \%$ in those with quiescent renal disease ${ }^{27}$. In past studies fetal losses ranged from 8 to $36 \%$, miscarriages between 4 and $31 \%$, and stillbirths or neonatal deaths between 4 and $23 \%{ }^{28,29}$. Treatment of flares $L N$ includes corticosteroids and hydroxychloroquine. Renal remission defined as follows stable renal function and serum creatinine in the normal range or estimated glomerular filtration rate $>60 \mathrm{ml} / \mathrm{min} / 1.73 \mathrm{~m} 2$, urinary red cells $<5 /$ high-power field, proteinuria $<0.5 \mathrm{~g} /$ day and normal serum C3 levels for at least 12-18 months and not 6 months only as proposed by others ${ }^{30}$. Antiphospholipid antibodies are present in SLE patients in some publications and the association between these antibodies and fetal loss has been well demonstrated ${ }^{31,32}$. Mechanisms could include lack of implantation, placental vasoconstriction and thrombosis leading to either fetal growth restriction or death ${ }^{33}$.

Pregnancy in SLE patients should be considered as a high-risk pregnancy and conception should be planned, if possible during a quiescent period. Close monitoring for 
optimal disease control and multidisciplinary obstetrical care are necessary throughout the gestation period to increase the chances of a successful pregnancy ${ }^{34}$.

\section{b. Antiphospholipid syndrome}

The APS is a disorder of the immune system that is characterized by excessive clotting of blood and/or certain complications of pregnancy (premature miscarriages, stillbirth, preterm delivery, severe preeclampsia unexplained fetal death, or premature birth) and the presence of antiphospholipid antibodies (cardiolipin or lupus anticoagulant antibodies) in the blood ${ }^{35}$. The risks are determined by the level of antibodies in the blood: higher levels indicate a higher risk of thrombotic events or complications such as visual disturbances, deep vein thrombosis, heart attack and stroke, fetal or neonatal thrombosis, recurrent miscarriage, early delivery, oligohydramnios, prematurity, intrauterine growth restriction, fetal distress $^{36}$. Like other autoimmune disorders, APS does not have a known etiology. APS is a systemic autoimmune disease characterized by an adaptive immune response against self-PL-binding proteins ending in the production of specific autoantibodies and autoantibodies promote activation of endothelial cells, monocytes and platelets, causing an overproduction of tissue factor and thromboxane A237. These factors associated with the typical changes in the hemostatic system during normal pregnancy result in a hypercoagulable state.

The APS takes most commonly in young to middle-aged adults. It also can perform in children and the elderly. Among patients with SLE, the prevalence of antiphospholipid antibodies is high ranging from $12 \%$ to $30 \%$ for anticardiolipin antibodies and $15 \%$ to $34 \%$ for lupus anticoagulant antibodies. Overall anticardiolipin antibodies occur five times more often then lupus anticoagulant in patients with APS38. The prevalence of anticardiolipin antibodies and lupus anticoagulants in healthy populations has been reported to range between $1.0 \%$ and $5.6 \%$ and between $1.0 \%$ and $3.6 \%$, respectively 39 . Approximately $40 \%$ of patients with SLE are antiphospholipid antibodies ${ }^{40}$. APS is associated with recurrent miscarriage in early pregnancy and other complications of pregnancy including preterm labour, preeclampsia and low birth weight. Fetal death beyond the tenth week of gestation is the most typical obstetric complication of APS. It is thought that placental ischaemia due to thrombus in the placenta are the cause. Pregnancy with APS should be examined frequently during their pregnancy for evidence of thrombosis, preeclampsia or any decrease of fetal movement. During the first trimester levels of human chorionic gonadotropin are measured to determine the ongoing safety of the pregnancy. Ultrasound is carried out every 3-4 weeks from 18 weeks' onward to check fetal growth which can be adversely affected. The consensus was that treatment for APS pregnant patients should be low molecular weight heparin $(\mathrm{LMWH})$ and low dose aspirin (LDA). Patients with previous thromboses usually receive two injections per day. Unfractionated heparin or low molecular weight heparin could be substituted when appropriate because these agents do not cross the placenta and are considered the anticoagulant drugs of choice during pregnancy ${ }^{41}$. If possible, warfarin therapy should be avoided during pregnancy and therapy of warfarin is due to the necessary such as previous stroke or severe arterial thromboses. It should be avoided at least during the first trimester due to because of teratogenicity and from about 2 to 4 weeks before delivery to reduce risk of hemorrhagic complications ${ }^{42}$.

\section{c. Rheumatoid arthritis}

RA is a chronic systemic inflammatory disease of unknown etiology that primarily targets synovial tissues and characteristically peripheral joints are symmetrically inflamed leading to progressive destruction of articular structures usually accompanied by systemic symptoms. Systemic symptoms include low-grade fever, early morning stiffness, generalized afternoon fatigue and malaise, anorexia, weakness and Joint symptoms include pain, swelling, and stiffness. As many as $75 \%$ of women with rheumatoid arthritis experience improvement of both joint and extra-articular features during pregnancy ${ }^{43}$. RA is relatively common with a prevalence of slightly less than $1 \%$ in adults all over the world ${ }^{44}$. RA is more common than SLE with an incidence of from 2.5 to 
7 per 10,000 per year and a prevalence of approximately $1 \%$ in the adult U.S. population and RA is more common in women than men ${ }^{45}$.

The exact cause of RA is unknown but it results from a complex interaction between genes and environment. $T$ cells, B cells and the orchestrated interaction of proinflammatory cytokines play key roles in the pathophysiology of RA. Differentiation of native T cells into Th 17 cells results in the production of IL-17 a potent cytokine that promotes synovitis. B cells further the pathogenic process through antigen presentation and autoantibody and cytokine production. The release of cytokines such as TNF-U, IL-6 and IL-1 causes synovial inflammation and cytokines promote the systemic effects including fatigue, production of acute phase proteins, anaemia, osteoporosis cardiovascular diseases and depression ${ }^{46}$. Some studies have confirmed that spontaneously healing of RA during pregnancy and an increased risk of flare after delivery. Pregnancy not only mitigates disease activity in RA patients but also decreases the risk for RA onset and improvement usually begins during the first trimester and rheumatoid nodules may disappear ${ }^{43}$. Pregnancy related changes in circulating hormones may contribute to alterations in the immune system that may impact disease activity. In a study conducted by Barrett et al, $63 \%$ of the patients reported improvement in disease activity at the third trimester although only $16 \%$ were in remission ${ }^{47}$. However in a study conducted by de Man et al, the disease activity decreased during pregnancy but increased after delivery ${ }^{48} .90 \%$ of those who experience remission suffer postpartum exacerbations and there is an increased incidence of rheumatoid arthritis onset in the postpartum period ${ }^{49}$. The majority of patients with active disease will experience improvement or total remission in the second half of pregnancy and more than $50 \%$ of patients will flare postpartum ${ }^{50}$

The most commonly used medications who require continued treatment or experience a disease flare during pregnancy are glucocorticoids, nonsteroidal antiinflammatory drugs (NSAIDs), aspirin. Prednisone and prednisolone cross the placenta but appear in only small amounts in cord blood51. Glucocorticoid therapy during pregnancy may increase the risk of premature rupture of the membranes and intrauterine growth restriction ${ }^{52}$, hypertension, gestational diabetes, osteoporosis, infection $^{53}$. According to data from a meta-analysis of pregnancy in women taking glucocorticoids reported an overall odds ratio for bearing a child with cleft palate of $3.4^{54}$. NSAIDs generally fall into FDA Category $B$ and some of them can be used safely in the first part of pregnancy, although increased risk of first trimester miscarriage under NSAID treatment has been reported and most NSAIDs therefore receive an FDA Category $C$ labelling beyond 30 weeks of gestation ${ }^{55}$. Fetal and neonatal adverse effects affecting the brain, kidney, lung, skeleton, gastrointestinal tract and cardiovascular system have also been reported after prenatal exposure to NSAIDs. NSAIDs should be given in pregnancy only if the maternal benefits outweigh the potential fetal risks at the lowest effective dose and for the shortest duration possible ${ }^{56}$. The efficacy of low-dose aspirin for this indication is controversial, individual patient data from over 32,000 women and their babies showed that low-dose aspirin had no significant effect on the risk of bleeding events in either the women or their babies ${ }^{57}$. Third trimester use of these agents may pose greater risk because of high-dose aspirin therapy in the third trimester has the potential for causing premature closure of the ductus arteriosus; indomethacin and ibuprofen appear to have much stronger ductal effects than aspirin and high-dose aspirin mayhap increase the risk of fetal or neonatal bleeding ${ }^{58,59}$. Medications with a high risk of causing birth defects should be avoided during conception and pregnancy including methotrexate and leflunomide.

\section{d. Scleroderma}

Systemic sclerosis (SSc, scleroderma) is a systemic, inflammatory, autoimmune disease affecting the skin and viscera manifesting pathologically with microvascular lesions, perivascular infiltration by mononuclear cells and increased deposition of extracellular collagen and characterized by widespread vascular injury and progressive fibrosis of the skin and internal organs. The rarity of the disease as well as its propensity to appear in the early 1940s, explain the low frequency of concurrent sclero- 
derma and pregnancy60. Leukotrienes play an important role in the regulation of all the processes vital to the pathogenesis of SSc namely inflammation, vascular function and connective tissue remodeling ${ }^{61}$. Many factors can lead to immunologic system disturbances and vascular changes. The activated cells secrete a variety of substances and they cause to changes in the extracellular matrix compounds including fibronectin, proteoglycans and collagens ${ }^{62}$. Therefore the characteristic signs of the disease occur following widespread vascular injury and progressive fibrosis of the skin and internal organs ${ }^{63}$.

Incidence and prevalence may be underestimated because patients with early or atypical disease may be overlooked in surveys. Incidence of the disease estimated at 9-19 cases per million per year and prevalance estimated that 286 cases per million population in United States ${ }^{64}$. SSc is characterized by a progressive fibrosis of the skin, lungs, kidneys, gastrointestinal tract, blood vessels, heart. SSc is characterized by symptoms that occur frequently in the general population including fatigue, gastroesophageal reflux, raynaud phenomenon, musculoskeletal pain. Therefore it is important for primary care practitioners to be aware of scleroderma because early intervention can reduce morbidity and detect life-threatening complications. The most common symptoms and signs in patients with SSc are malaise, myalgias, Raynaud 's syndrome, swelling of the distal extremities with gradual thickening of the skin of the fingers, fatigue, arthralgias and less commonly noted symptoms were breathlessness, upset stomach, sore eyes, depression, nausea and weight loss65. The other clinical manifestations in patients with SSc are esophageal dysfunction, cardiac arrhythmias, pericarditis, pulmonary hypertension, sudden renal disease and hypertension ${ }^{66}$.
All patients who become pregnant during this high-risk time should be monitored extremely carefully. The high risk of premature and small infants may be minimized with specialized obstetric and neonatal care, however. Other pregnancy problems may not be unique to scleroderma but because it is a chronic illness any complication carries higher risks for both mother and child ${ }^{67}$. The current retrospective studies show that despite an increased frequency of prematurity and small for gestational age infants overall maternal and neonatal survival is good ${ }^{68}$. Hence, at present with close monitoring and appropriate therapy most scleroderma patients can sustain a successful pregnancy. The worst life-threatening complication of a pregnancy is scleroderma renal crisis: despite the fact that ACE inhibitors are associated with congenital abnormalities and are relatively contraindicated in pregnancy in this case their use is recommended $^{69}$. Hydroxychloroquine and low doses of steroids may be safely used. In order to minimize risks a multidisciplinary approach is necessary to suggest the best timing for a pregnancy and provide adequate supportive treatment to SSc patients during the pregnancy ${ }^{70}$.

\section{Conclusion}

Pregnancies in women with rheumatic diseases require a multidisciplinary, coordinated approach before during and after pregnancy. During pregnancy it is essential to monitor all aspects of disease activity (renal involvement), pregnancy complications (intrauterine growth retardation, thromboembolic disease, preeclampsia et al.). Post-partum flare is common in all the rheumatic diseases. 


\section{References}

1. Jain V, Gordon C. Managing pregnancy in inflammatory rheumatological diseases. Arthritis Res Ther. 2011 Feb 25;13(1):206 .

2. Chang J, Streitman D. Physiologic adaptations to pregnancy. Neurol Clin. 2012 Aug;30(3):781-9

3. Lund CJ, Donovan JC. Blood volume during pregnancy. Significance of plasma and red cell volumes. Am J Obstet Gynecol 1967;98(3):394-403.

4. Marik P, Plante LA. Venous thromboembolic disease and pregnancy. N Engl J Med 2008;359(19):2025-33.

5. Heckman JD, Sassard, R. Current concepts review. Musculoskeletal considerations in pregnancy. J. Bone Joint Surg. Am 1994;76(11):1720-1730.

6. Van den Broe NR, Letsky EA. Pregnancy and the erythrocyte sedimentation rate. BJOG 2001; 108(11):1164-1167.

7. Cutolo M, Capellino S, Straub RH. Estrogens in rheumatic diseases: friend or foe? Rheumatology 2008; 47(3): 2-5.

8. Doria A, laccarino L, Arienti S, Ghirardello A, Zampieri S, Rampudda $M E$, et al. Th2 immune deviation induced by pregnancy: the two faces of autoimmune rheumatic diseases. Reprod Toxicol 2006; 22(2): 234-41.

9. Ostensen M, Förger F, Villiger PM. Cytokines and pregnancy in rheumatic disease. Ann N Y Acad Sci 2006;1069:353-63.

10. Lawrence RC, Helmick CG, Arnett FC, Deyo RA, Felson DT, Giannini EH, et al. Estimates of the prevalence of arthritis and selected musculoskeletal disorders in the United States. Arthritis Rheum 1998;41(5):778-99.

11. Rivest C, Lew RA, Welsing PM, Sangha O, Wright EA, Roberts $W N$, et al. Association between clinical factors, socioeconomic status, and organ damage in recent onset systemic lupus erythematosus. J Rheumatol 2000;27(3):680-4.

12. Skomsvoll JF, Aasarød K, Salvesen KA, Hoff M, Wallenius M, Rødevand $\mathrm{E}$, et al. Systemic lupus erythematosus and pregnancy. Tidsskr Nor Laegeforen 2007;127(6):725-9.

13. Lockshin MD, Reinitz $E$, Druzin ML, Murrman M, Estes D. Lupus pregnancy. Case-control prospective study demonstrating absence of lupus exacerbation during or after pregnancy. Am J Med 1984;77(5):893-8.

14. Ruiz-Irastorza G, Lima F, Alves J, Khamashta MA, Simpson J, Hughes GR, et al. Increased rate of lupus flare during pregnancy and the puerperium: a prospective study of 78 pregnancies. $\mathrm{Br} \mathrm{J}$ Rheumatol 1996;35(2):133-8.

15. Ostensen M, Brucato A, Carp H, Chambers C, Dolhain RJ, Doria $A$, et al. Pregnancy and reproduction in autoimmune rheumatic diseases. Rheumatology (Oxford) 2011;50(4):657-64.

16. Clowse ME, Jamison M, Myers $E$, James $A H$. A national study of the complications of lupus in pregnancy. Am J Obstet Gynecol 2008;199(2):127.e1-6

17. Mok CC, Wong RW. Pregnancy in systemic lupus erythematosus. Postgrad Med J 2001;77:157-65

18. Moroni G, Ponticelli C. The risk of pregnancy in patients with lupus nephritis. J Nephrol 2003;16:161-167.

19. Urowitz MB, Gladman DD, Farewell VT, Stewart J, McDonald J. Lupus and pregnancy studies. Arthritis Rheum 1993;36:1392-7.

20. Gayed M, Gordon C. Pregnancy and rheumatic diseases. Rheumatology 2007;46:1634-1640.

21. Shehata N, Burrows R, Kelton JG. Gestational thrombocytopenia. Clin Obstet Gynecol 1999;42:327-34

22. Magann EF, Martin JN Jr. Twelve steps to optimal management of HELLP syndrome. Clin Obstet Gynecol 1999;42:532-50.

23. Levy JA, Murphy LD. Thrombocytopenia in pregnancy. J Am Board Fam Pract 2002;15(4):290-7.

24. Chakravarty EF, Nelson L, Krishnan E. Obstetric hospitalizations in the United States for women with systemic lupus erythematosus and rheumatoid arthritis. Arthritis Rheum 2006;54:899-907.

25. Heard AR, Dekker GA, Chan A, Jacobs DJ, Vreeburg SA, Priest KR. Hypertension during pregnancy in South Australia, Part 1: pregnancy outcomes. Australian and New Zealand Journal of Obstetrics and Gynaecology 2004;44(5):404-409.

26. Mustafa R, Ahmed S, Gupta A, Venuto RC. A comprehensive review of hypertension in pregnancy. J Pregnancy 2012; 2012:105918.

27. Cervera R, Font J, Carmona F, Balasch J. Pregnancy outcomes in systemic lupus erythematosus: good news for the new millennium. Autoimmun Rev 2002;1: 354-359.

28. Mok CC, Wong RWS. Pregnancy in systemic lupus erythematosus. Postgrad Med J 2001;77: 157-165.

29. Moroni G, Ponticelli C. The risk of pregnancy in patients with lupus nephritis. J Nephrol 2003; 16:161-167.

30. Kong NC. Pregnancy of a lupus patient-a challenge to the nephrologist. Nephrol Dial Transplant 2006;21: 268-272.

31. Cortes-Hernandez J, Ordi-Ros J, Paredes F, Casellas M, Castillo F, Vilardell-Tarres M. Clinical predictors of fetal and maternal outcome in systemic lupus erythematosus: a prospective study of 103 pregnancies. Rheumatology (Oxford) 2002;41:643-50.

32. Carmona F, Font J, Cervera R, Munoz F, Cararach V, Balasch J. Obstetrical outcome of pregnancy in patients with systemic Lupus erythematosus. A study of 60 cases. Eur J Obstet Gynecol 
Reprod Biol 1999;83:137-42

33. Packham DK, Lam SS, Nicholls K, Fairley KF, Kincaid-Smith PS. Lupus nephritis and pregnancy. Q J Med 1992;83:315-24.

34. Yan Yuen S, Krizova A, Ouimet JM, Pope JE. Pregnancy Outcome in Systemic Lupus Erythematosus (SLE) is Improving: Results from a Case Control Study and Literature Review. Pregnancy Outcome in Systemic Lupus Erythematosus (SLE) is Improving: Results from a Case Control Study and Literature Review. Open Rheumatol J. 2008;2:89-98.

35. Mialdea M, Sangle SR, D'Cruz DP. Antiphospholipid (Hughes) syndrome: beyond pregnancy morbidity and thrombosis. Journal of Autoimmune Diseases 2009;19:6.

36. Branch DW, Khamashta MA. Antiphospholipid syndrome: obstetric diagnosis, management, and controversies. Obstet Gynecol. 2003 Jun;101(6):1333-44.

37. Meroni PL. Pathogenesis of the antiphospholipid syndrome: an additional example of the mosaic of autoimmunity. J Autoimmun 2008;30(1-2):99-103.

38. Gezer S. Antiphospholipid syndrome. Dis Mon 2003;49(12):696741.

39. de Groot PG, Lutters B, Derksen RH, Lisman T, Meijers JC, Rosendaal FR. Lupus anticoagulants and the risk of a first episode of deep venous thrombosis.J Thromb Haemost. 2005;3(9):19937.

40. Mok CC, Tang SS, To $\mathrm{CH}$, Petri M. Incidence and risk factors of thromboembolism in systemic lupus erythematosus: a comparison of three ethnic groups. Arthritis Rheum 2005;52(9):2774-82.

41. Tincani A, Branch W, Levy RA, Piette JC, Carp H, Rai RS, et al. Treatment of pregnant patients with antiphospholipid syndrome. Lupus 2003;12(7):524-9.

42. Abadi S, Einarson A, Koren G. Use of warfarin during pregnancy. Can Fam Physician. 2002;48:695-7.

43. Nelson JL, Ostensen M. Pregnancy and rheumatoid arthritis. Rheum. Dis. Clin. North Am 1997:23:195-212.

44. James R. O'Dell. Rheumatoid Arthritis. In: Lee Goldman, Andrew I. Schafer (eds), Goldman's Cecil Medicine, 24th ed. Philadelphia: Saunders, An Imprint of Elsevier; 2012. p.1681.

45. Jeff M. Denney, Troy Flint Porter, D. Ware Branch. High Risk Pregnancy. Autoimmune Diseases. In: David James, Philip J. Steer, Carl P. Weiner, Bernard Gonik (eds), High Risk Pregnancy, 4th ed. St. Louis: Saunders, An Imprint of Elsevier; 2010. p.783.

46. Choy E.Understanding the dynamics: pathways involved in the pathogenesis of rheumatoid arthritis. Rheumatology (Oxford) 2012;51:v3-v11.

47. Barrett JH, Brennan P, Fiddler M, Silman AJ. Does rheumatoid arthritis remit during pregnancy and relapse postpartum? Results from a nationwide study in the United Kingdom performed prospectively from late pregnancy. Arthritis Rheum Jun 1999;42(6):1219-27.

48. de Man YA, Dolhain RJ, van de Geijn FE, Willemsen SP, Hazes JM. Disease activity of rheumatoid arthritis during pregnancy: results from a nationwide prospective study. Arthritis Rheum 2008;59(9):1241-8.

49. Daghni Rajasingam, Catherine Nelson-Piercy. Connective Tissue Diseases in Pregnancy. In: Martin Black, Christina M. AmbrosRudolph, Libby Edwards, Peter J. Lynch (eds), Obstetric and Gynecologic Dermatology, 3rd ed. Mosby, An Imprint of Elsevier, 2008. p.99

50. Ostenson M. Pregnancy in patients with a history of juvenile rheumatoid arthritis. Arthritis Rheum 1991;34:881-887.

51. Rennick GJ. Use of systemic glucocorticosteroids in pregnancy: be alert but not alarmed. Australas J Dermatol 2006:47(1):34-6.

52. Guller S, Kong L, Wozniak R, Lockwood CJ. Reduction of extracellular matrix protein expression in human amnion epithelial cells by glucocorticoids: a potential role in preterm rupture of the fetal membranes. J Clin Endocrinol Metab 1995;80:2244-50

53. Østensen $M$, Khamashta $M$, Lockshin $M$, Parke $A$, Brucato $A$, Carp $\mathrm{H}$, et al. Anti-inflammatory and immunosuppressive drugs and reproduction. Arthritis Res Ther 2006;8:209.

54. Park-Wyllie L, Mazzotta P, Pastuszak A, Moretti ME, Beique $L$, Hunnisett $L$, et al. Birth defects after maternal exposure to corticosteroids: prospective cohort study and meta-analysis of epidemiological studies. Teratology 2000;62:385-392.

55. Hazes JM, Coulie PG, Geenen V, Vermeire S, Carbonnel F, Louis E, et al. Rheumatoid arthritis and pregnancy: evolution of disease activity and pathophysiological considerations for drug use. Rheumatology (Oxford) 2011;50(11):1955-68

56. Antonucci R, Zaffanello M, Puxeddu E, Porcella A, Cuzzolin L, Pilloni MD, et al. Use of non-steroidal anti-inflammatory drugs in pregnancy: impact on the fetus and newborn. Curr Drug Metab 2012;13(4):474-90.

57. Askie LM, Duley L, Henderson-Smart DJ, Stewart LA; PARIS Collaborative Group. Antiplatelet agents for prevention of preeclampsia: a meta-analysis of individual patient data. Lancet 2007;369(9575):1791-8.

58. Koren G, Florescu A, Costei AM, Boskovic R, Moretti ME. Nonsteroidal antiinflammatory drugs during third trimester and the risk of premature closure of the ductus arteriosus: a metaanalysis. Ann Pharmacother 2006;40:824-9.

59. Ostensen M, Ramsey-Goldman R. Treatment of inflammatory 
rheumatic disorders in pregnancy: what are the safest treatment options? Drug Saf 1998;19:389-410.

60. Lidar M, Langevitz P. Pregnancy issues in scleroderma. Autoimmun Rev 2012;11(6-7):A515-9.

61. Chwiesko-Minarowska S, Kowal K, Bielecki M, Kowal-Bielecka $\mathrm{O}$. The role of leukotrienes in the pathogenesis of systemic sclerosis. Folia Histochem Cytobiol 2012;50(2):19348.

62. Sakkas LI. New developments in the pathogenesis of systemic sclerosis. Autoimmunity 2005;38(2):113-6.

63. Betty Diamond. Immunologic Mechanisms in Disease. In: William E.Paul (eds), Fundamental Immunology Sixth Edition. Philadelphia: Lippincott Williams \& Wilkins, a Wolters Kluwer, 2008. p.1320-1321.

64. John Varga, Christopher P. Denton. Scleroderma, inflammatory myopathies, and overlap syndromes. In: Ralph C. Budd, Edward D. Harris Jr, lain B. Mclnnes, Shaun Ruddy (eds), Kelley's Textbook of Rheumatology, 8th ed. Philadelphia: W. B. Saunders Company, 2008. p.1312.
65. Nona T. Colburn. Systemic Sclerosis and Related Syndromes. In: Nona T. Colburn (eds), Review of Rheumatology. New York: Springer-Verlag London Limited, 2012. P.363-389.

66. Robert S. Porter and Justin L. Kaplan. Autoimmune Rheumatic Disorders. In: Robert S. Porter and Justin L. Kaplan (eds), The Merck Manual Of Diagnosis and Therapy, 19th ed. . West Point, PA : Merck Sharp \& Dohme Corp., A Subsidiary of Merck \& Co., Inc, 2011. p.309-312.

67. Steen VD. Scleroderma and pregnancy Rheum Dis Clin North Am 1997;23(1):133-47.

68. Lidar M, Langevitz P. Pregnancy issues in scleroderma. Autoimmun Rev 2012;11(6-7):A515-9.

69. Miniati I, Guiducci S, Mecacci F, Mello G, Matucci-Cerinic M. Pregnancy in systemic sclerosis. Rheumatology (Oxford) 2008;47 Suppl 3:iii16-8.

70. Bérezné A, Mouthon L.Pregnancy in systemic sclerosis. Presse Med 2008:37(11):1636-43. 